\title{
Neural responses to visual food cues according to weight status: a systematic review of functional magnetic resonance imaging studies
}

\author{
Kirrilly M. Pursey ${ }^{1}$, Peter Stanwell ${ }^{2}$, Robert J. Callister ${ }^{3}$, Katherine Brain $^{1}$, Clare E. Collins ${ }^{1}$ and \\ Tracy L. Burrows ${ }^{1}$ * \\ 1 School of Health Sciences, Priority Research Centre for Physical Activity and Nutrition, University of Newcastle, Callaghan, NSW, Australia \\ ${ }^{2}$ School of Health Sciences, Priority Research Centre for Translational Neuroscience and Mental Health, University of Newcastle, Callaghan, NSW, Australia \\ ${ }^{3}$ School of Biomedical Sciences and Pharmacy, Priority Research Centre for Translational Neuroscience and Mental Health, University of Newcastle, Callaghan, \\ NSW, Australia
}

\section{Edited by:}

Jens Blechert, University of Salzburg,

Austria

\section{Reviewed by:}

Sylvia Richter, Paris-Lodron-University Salzburg, Austria

Hubert Preissl, University of Tübingen, Germany

\section{*Correspondence:}

Tracy L. Burrows, School of Health

Sciences, Priority Research Centre for Physical Activity and Nutrition,

University of Newcastle, University

Drive, Callaghan, NSW 2308, Australia

e-mail: tracy.burrows@

newcastle.edu.au
Emerging evidence from recent neuroimaging studies suggests that specific food-related behaviors contribute to the development of obesity. The aim of this review was to report the neural responses to visual food cues, as assessed by functional magnetic resonance imaging (fMRI), in humans of differing weight status. Published studies to 2014 were retrieved and included if they used visual food cues, studied humans $>18$ years old, reported weight status, and included fMRI outcomes. Sixty studies were identified that investigated the neural responses of healthy weight participants $(n=26)$, healthy weight compared to obese participants $(n=17)$, and weight-loss interventions $(n=12)$. High-calorie food images were used in the majority of studies ( $n=36)$, however, image selection justification was only provided in 19 studies. Obese individuals had increased activation of reward-related brain areas including the insula and orbitofrontal cortex in response to visual food cues compared to healthy weight individuals, and this was particularly evident in response to energy dense cues. Additionally, obese individuals were more responsive to food images when satiated. Meta-analysis of changes in neural activation post-weight loss revealed small areas of convergence across studies in brain areas related to emotion, memory, and learning, including the cingulate gyrus, lentiform nucleus, and precuneus. Differential activation patterns to visual food cues were observed between obese, healthy weight, and weight-loss populations. Future studies require standardization of nutrition variables and fMRI outcomes to enable more direct comparisons between studies.

Keywords: functional magnetic resonance imaging, fMRI, food cues, visual, obesity, weight status

\section{INTRODUCTION}

The prevalence of obesity is increasing rapidly (1) with $33.6 \%$ of people in the United States classified as overweight and $34.9 \%$ classified as obese in 2011-2012 (2). Obesity increases the risk for a variety of lifestyle diseases including cardiovascular disease, hypertension, diabetes, and some cancers (3), as well as reduced quality of life (4). To minimize the substantial economic and health burden of obesity, numerous approaches have been used to target overweight and obese individuals to facilitate weight loss, including lifestyle and surgical interventions (5). Weight-loss interventions focusing on behavioral changes such as dietary modifications and increased physical activity are commonly used; however, these have demonstrated variable effects on weight loss and its long-term maintenance (6). Recently, there has been interest in the possible role that neural mechanisms play in the development and maintenance of obesity. In addition, increasing attention has been given to investigating the impact these neural mechanisms may have on weight loss and maintenance.

It has been suggested that neural responses to specific foods parallel those that are observed in drug dependence and chronic addiction $(7,8)$. To date, neuroimaging techniques, such as functional magnetic resonance imaging (fMRI), have provided a technique to report the activation of reward-related brain regions in response to food. Visual and olfactory food cues as well as actual food intake have been shown to activate similar brain regions to that of illicit drugs (9-13) in susceptible individuals $(14,15)$. These fMRI studies provide new insights into the neurobiology of eating behavior and food-cue responsivity, and suggest that abnormal eating behaviors such as overeating in obesity involve alterations in an individual's neurocircuitry $(9,10)$. This could have a significant impact on weight status, and potentially contribute to the current prevalence of obesity. Additionally, alterations in neurocircuitry could provide an explanation for some of the lack of effectiveness of weight-loss interventions, as well as maintenance of weight loss in susceptible individuals (16-18).

Many fMRI studies have attempted to identify the neural correlates of eating behavior that could potentially lead to obesity. A great deal of heterogeneity, however, is evident in study design and methodological techniques across the available studies to date (19-21). Previous literature on the neural processing of visual food 
cues has identified alterations in limbic, paralimbic, and frontal brain circuits. These brain areas are associated with emotional salience, memory, reward, and cognitive and visual processing. Furthermore, motivational state, weight status, and energy density of presented foods have been reported to affect neural responses (20-24). Reviews of the literature investigating neural responses to food cues to date have included individuals with eating disorders or used multiple stimuli modalities such as taste. These approaches may affect neural responses to food cues (20) or recruit multiple anatomical centers in the brain $(21,25)$.

Specific meta-analysis has emerged as a method to overcome the heterogeneity in fMRI studies. Activation likelihood estimation (ALE) meta-analysis is a technique that integrates findings of fMRI experiments to identify common or divergent activation patterns across a range of studies, using standardized brain coordinates. Existing ALE meta-analyses assessing neural activation of healthy weight individuals to visual food cues have shown that the hunger-state and salience of the presented food items alters activity in brain regions associated with arousal, reward processing, attention, visual processing, and memory of previous food experiences (22). Subsequent meta-analyses have found that overweight and obese individuals have altered activity in brain regions associated with related-cue processing, decision making, anticipation, caloric appraisal, arousal, and memory $(23,24)$.

Currently, the process by which the human brain integrates food signals to produce maladaptive eating behaviors such as overeating in obesity is largely unknown and warrants further investigation. No studies to date have systematically reviewed the neural responses to visual food cues across all weight categories in individuals who do not have a diagnosed history of abnormal eating behavior such as an eating disorder. Additionally, no studies have systematically reviewed published studies or applied metaanalytical techniques to neural responses to visual food cues preand post-weight loss. This is important as it could have implications for the development of more effective weight-loss treatments and maintenance of lost weight.

The aim of this systematic review was to examine published literature related to neural activation, as measured by fMRI, in response to visual food cues by weight status. The primary aim of the review was to determine whether differential neural responses are observed when viewing visual food cues based on body mass index (BMI) category. A secondary aim of the review was to determine whether different neural activation patterns are observed in response to viewing food compared to non-food cues in individuals before and after weight loss.

\section{MATERIALS AND METHODS}

A review was undertaken to identify published studies in the English language that used fMRI as a primary outcome measure of neural responses to visual food cues from 1973 to March 2014. This process is outlined in Figure 1.

Initially, electronic databases were searched including: MEDLINE, The Cochrane Library, EMBASE (Excerpta Medica Database), CINAHL (Cumulative Index to Nursing and Allied Health), Informit Health Collection, Proquest, Web of Science, Scopus, and PsycINFO. A pre-determined list of keyword search terms was informed and compiled from a preliminary search of the literature

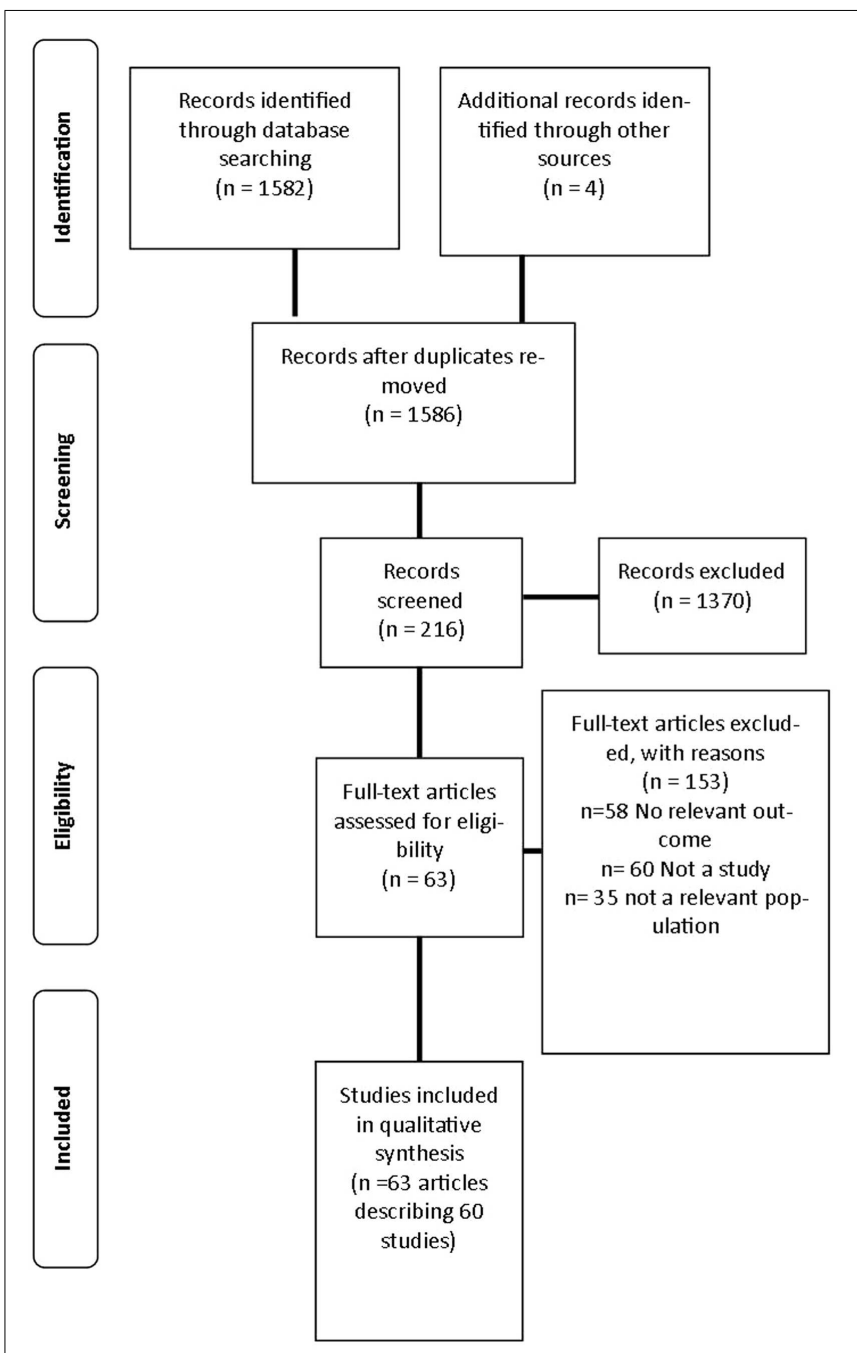

FIGURE 1 | Flow diagram of studies included in the review.

and expanded medical subject headings (MeSH). Keywords were used individually and in combination and included: fMRI, blood oxygen level dependent (BOLD), functional imaging, BOLD signal, BOLD effect, oxyhemoglobin, and deoxyhemoglobin, reward, overeating, addiction, process addiction, food addiction, binge, craving, and dopamine. In addition, electronic searches were supplemented by systematically checking reference lists of relevant publications.

Following the removal of duplicate references, titles and abstracts of identified studies were assessed by two independent reviewers (KP and PS). A predetermined inclusion criterion was applied to determine the study's eligibility in the review. Studies were included if they investigated an adult population ( $>18$ years of age), used visual food cues, reported weight status, and included fMRI as an outcome measure. Studies reporting a range of BMI categories were included to examine the relationship between neural activation and weight status. Healthy weight individuals were included in the review to act as a comparative group for examining brain activation to visual food cues across all weight 
status categories. Studies involving participants with a previous or current eating disorder including anorexia nervosa, bulimia nervosa, and binge-eating disorder were excluded, as these participants may have variable responses to food cues that could be attributed to the diagnosis of an eating disorder. Additionally, children or adolescents ( $<18$ years of age); participants with mental and neurological disorders including Prader-Willi Syndrome; pharmacological interventions and fMRI investigations into food intake alone were excluded in keeping with studies included in this review (26-29). If a study included a population meeting the exclusion criteria but reported fMRI outcomes separately for healthy weight and overweight/obese participants, only data on the healthy weight and overweight/obese population was reported in the review. Articles were retrieved for all studies that met the inclusion criteria. If eligibility was unclear, the article was retrieved for further clarification.

Studies were quality checked by two independent reviewers using a standardized 10-question tool (30). The assessed quality criteria included the source of funding, method of sample selection, intervention description, study blinding, and statistical analysis. Four of the quality criteria were designated as "important" and needed to be met to receive a high quality rating. These included: sample selection, comparability of study groups, intervention description, and validity and reliability of outcome measures. An overall classification quality was assigned to each study. Studies were classified as positive quality if $>5 / 10$ criteria were satisfied and all important criteria were met. If the majority of criteria were satisfied but at least one of the important criteria was not met, the study was classified as neutral quality. If the majority of the criteria $(>5 / 10)$ or important questions $(\geq 2 / 4)$ were not satisfied, the study was classified as negative quality. Criteria were classified as unclear if the reviewers could not determine whether criteria were met from the detail provided in the published article. Additionally, quality-related fMRI outcomes such as cluster size and volume were extracted and reported in the review. No studies were excluded based on quality ratings. Data were extracted using standardized tables developed for the review. In cases of uncertainty of a study's inclusion, quality assessment or data extraction were resolved by the consultation of a third independent reviewer until consensus was reached.

Studies were grouped and analyzed by BMI using the World Health Association (WHO) classification, i.e., underweight $\left(<18.49 \mathrm{~kg} / \mathrm{m}^{2}\right)$, healthy weight $\left(18.00-24.99 \mathrm{~kg} / \mathrm{m}^{2}\right)$, overweight $\left(25.00-29.99 \mathrm{~kg} / \mathrm{m}^{2}\right)$, or obese $\left(>30.00 \mathrm{~kg} / \mathrm{m}^{2}\right)(31)$. Four groups were created for analysis including: (1) studies that compared healthy weight individuals to overweight/obese individuals; (2) studies investigating individuals pre- and post-weight loss; (3) studies of healthy weight individuals only; (4) studies of overweight/obese individuals only. For the purposes of this study, individuals classified as underweight using the WHO cut points were included in the healthy weight category. Additionally, in studies where BMI spanned a number of categories, the mean BMI was used to classify the study into a specific weight category.

\section{META-ANALYSIS}

To determine the convergence of reported coordinates across studies investigating changes in neural responses pre- and post-weight loss, a meta-analysis was undertaken using the Brainmap GingerALE software ${ }^{1}$. The inclusion criteria for the meta-analysis were identical to the systematic review criteria. In addition, studies were required to report fMRI outcomes of changes in neural activation to visual food cues pre- to post-weight loss (surgical and behavioral) using either Talairach or Montreal Neurological Institute (MNI) coordinates. Only articles reporting whole brain analysis results were included as region of interest analysis is known to inflate activation findings (32). Papers reporting Talairach coordinates were converted to MNI coordinates prior to analysis using the GingerALE software.

Activation likelihood estimation meta-analysis applies a statistical modeling technique (32) that uses reported brain coordinates and adjusts for between-subject and between-template variance to generate a 3-dimensional Gaussian kernel. Subsequently, a modeled activation (MA) map is created and individual maps are combined to generate an experimental ALE map. The experimental map is tested against an ALE null distribution map, representing the null hypothesis that there is random variation between activation across the meta-analyzed studies, when the withinstudy variation remains fixed. A random-effects model is applied, which assumes convergence between different studies that is above chance.

A statistical threshold of $P<0.05$ False Discovery Rate (FDR), corrected for multiple comparisons and a minimum cluster size of $100 \mathrm{~mm}^{3}$ was set. This is consistent with previous meta-analyses in this area to control for publication bias with respect to the reporting of foci (22-24). Results of meta-analyses are presented using the Mango software package ${ }^{2}$.

\section{RESULTS}

The search strategy identified 1586 articles, 216 articles were screened for inclusion with 64 articles describing 60 studies included in the final analysis (26-29, 33-92) as described in Table S1 in Supplementary Material. The primary reasons for exclusion were: the article did not meet inclusion criteria for study design $(n=60)$; no relevant outcome was studied $(n=58)$; and the study investigated a population not specified in the inclusion criteria $(n=35)$. Three additional studies were excluded as they did not report BMI or weight status of participants (93-95).

A total of 1565 participants were included across the studies (mean 26, range 5-100). Age ranged from 18-66 years with the most commonly studied age group being $18-35$ years olds $(n=42$ studies) $(26,28,29,33-43,45-47,60-66,68-72,74-86,89,91$, 92). Participants were predominantly female and right handed with 26 of the studies exclusively recruiting females $(26,29,33$, $43,45-48,56-59,65,66,70-72,75,76,78-81,83-85,90-92)$. The majority of studies were published post $2009(n=53,83 \%)$ and used a within participants cross-over design $(n=25,42 \%)$. No randomized control trials were retrieved by the search criteria.

Seventeen of the included studies compared both obese and healthy weight participants in the same study (26-28, 33-47). Twelve reported outcomes from pre- to post-weight loss (48-59). Methods of weight loss included bariatric surgery $(n=7)(48,49$,

\footnotetext{
${ }^{1}$ http://www.brainmap.org/index.html

${ }^{2}$ http://rii.uthscsa.edu/mango/
} 
$52,53,57-59)$ and behavioral nutrition and lifestyle interventions $(n=5)(51,53-56)$ with follow up in these studies ranging from one month to twelve months. Weight loss ranged from 3.4-25\% of original body mass in these studies. Five of the included studies were exclusively conducted in overweight or obese participants $\left(\mathrm{BMI} \geq 25.00 \mathrm{~kg} / \mathrm{m}^{2}\right)(88-92)$ and 26 studied individuals with a mean BMI in the healthy weight range $\left(\mathrm{BMI}<25.00 \mathrm{~kg} / \mathrm{m}^{2}\right)(29$, 60-87). However, eight of the healthy weight studies included participants with BMI's spanning from the underweight category to the overweight/obese category $(29,67,69,74,78,79,81,82)$.

As outlined in Table S1 in Supplementary Material, 30 studies $(50 \%)$ used participants who were fasted prior to fMRI scans (range 2-24h) (26-29, 40-42, 44-46, 50, 51, 54, 55, 64, 67, 73, $75,77-82,86,87,90-92)$. Seven studied satiated participants (43, $47,48,58-60,76,83-85)$ and 23 investigated neural responses in both fasted and satiated conditions $(27,33-39,52,53,56,57,61-$ $63,65,66,68-72,74,88,89)$. The most common variables other than fMRI assessed in the included studies were hunger $(n=34$ studies), appetite ( $n=10$ studies), and liking ratings of presented foods ( $n=10$ studies).

Food images used in the studies were described by their authors as "high-calorie" foods in 36 of the 60 studies $(26,28,29,35,38,40$, 41, 43-50, 54-61, 64-66, 68, 69, 71, 76, 78-81, 83-88, 90-92) and included foods such as chocolate, chips, and hamburgers. Foods described as "low-calorie" foods were used in 32 studies $(28,35$, 38, 40, 42, 44, 46-48, 50, 54-61, 64-66, 68, 69, 71, 78-81, 83-88, 90-92) and included foods such as fruit and vegetables. The actual calorific values for foods were only reported in seven of the studies $(35,47,57-59,78,88)$. No studies reported the use of a dietitian or nutritionist in the selection or classification of foods. Fifteen studies used foods based on the appeal and salience of the food (e.g. "hedonic," "palatable," and "appetizing”) (27, 33, 34, 41, 43, 52, 62, 63, 70, 73, 75, 77-79, 81, 82). Food images were selected using pilot ratings of palatability, perceived calorific value, and recognizability of presented images in only 19 studies $(27,36,37$, 40, 43, 44, 52, 53, 61, 68, 70, 71, 74, 77, 79-82, 86). Control images were used in the majority $(n=48)$ of the included studies and varied greatly, including images of cars, office equipment, landscapes, and blurred images.

Block design was used in 38 of the studies $(27,28,34,35,37-$ 42 , 44, 46-53, 55, 56, 60-64, 67-69, 71-73, 81-85, 87-89, 91, 92) and a $3 \mathrm{~T}$ magnet was used most commonly to acquire imaging data $(n=43)(26,28,29,34,36,37,39-46,49-53,55,56$, 60-63, 65-69, 71-73, 75, 76, 78-82, 87, 89-92) (Table S2 in Supplementary Material). The imaging plane most commonly used to acquire images of the brain was the transverse plane parallel to the anterior commissure posterior commissure line (AC-PC line) $(n=18)$. The method of reporting fMRI results was variable across the range of studies. All studies excluding two reported Talairach or MNI coordinates, but only 32 (53\%) studies reported cluster size or volume of activation $(35,38,39,41,43,44,46,49,50,52$, 53, 55-59, 62-64, 66-69, 72, 74, 77, 78, 81, 84-86, 88, 91).

HEALTHY WEIGHT COMPARED TO OVERWEIGHT/OBESE PARTICIPANTS Across studies comparing overweight and obese participants to healthy weight controls, overweight/obese individuals had greater brain activity to foods compared to non-foods in areas associated with a variety of functions in the context of food-cue processing (96). This included areas associated with reward processing [insula $(26,33,41,43,47)$, orbitofrontal cortex (OFC) $(26,28$, $43)$ ], reinforcement and adaptive learning [amygdala $(27,28,33$, $43)$, putamen $(28,41,47)$, OFC $(26,28,43)]$, emotional processing [insula $(26,33,41,43,47)$, amygdala $(27,28,33,43)$, cingulate gyrus $(44,45)]$, recollective, and working memory [amygdala (27, $28,33,43)$, hippocampus $(27,28,33,45)$, thalamus $(33,41)$, posterior cingulate cortex $(27,47)$, caudate $(28,45,47)]$, executive functioning [prefrontal cortex (PFC) $(28,43)$, caudate $(28,45$, $47)$, cingulate gyrus $(44,45)]$, decision making $[$ OFC $(26,28,43)$, PFC $(28,43)$, thalamus $(33,41)]$, and visual processing [thalamus $(33,41)$, fusiform gyrus $(27,43)]$. Additionally, obese individuals displayed greater activation to food cues in areas involved in motor learning and coordination such as hand-to-mouth movements and swallowing [insula $(26,33,41,43,47)$, putamen $(28,41$, $47)$, thalamus $(33,41)$, caudate $(28,45,47)]$ as well as risk aversion [inferior frontal gyrus $(41,44)$ ]. These increases in brain activity were particularly evident in response to high-calorie foods compared to low-calorie foods. When satiated, increased activity was observed in obese compared to healthy weight individuals in areas involved in decision making [PFC $(34,39)$, OFC, and caudate $(38)]$, reward anticipation [anterior cingulate $(33,38)$ and OFC $(33,38)$ ] as well as emotional processing [insula $(33,37)$, caudate (38), and amygdala (33)]. Significant correlations between BMI and activation were reported in three studies $(37,44,47)$.

\section{WEIGHT CHANGE INTERVENTIONS}

In three studies using a nutrition and lifestyle intervention, brain activation at the commencement of the intervention was associated with degree of weight-loss success and maintenance. This included areas associated with reward processing and anticipation [insula, anterior cingulate cortex, nucleus accumbens (55), and the OFC (56)], decision making [PFC (54) and OFC (56)], and impulsivity [nucleus accumbens and the anterior cingulate cortex (55)]. Participants who had successfully lost and maintained weight-loss displayed differential neural responses to food cues to those of healthy weight participants in areas involved in emotion, memory, and visual processing [cingulate gyrus, parietal cortex (51)], and to that of obese participants in regions associated with emotion, impulse control, and reward-based learning [PFC and the anterior cingulate (50)].

In studies reporting pre- to post-bariatric surgery outcomes, reductions in activity were reported in the insula and putamen. These areas are implicated in interoceptive processing $(52,58)$ and reinforcement learning $(57,59)$, respectively. Further, activation of the hypothalamus, which regulates hunger and subsequent food intake, following gastric bypass surgery resembled the responses of healthy weight individuals more closely than responses of obese individuals. More successful weight loss in gastric bypass surgery was associated with increased baseline neural activity of the dorsolateral PFC (49) and unique changes in activity were found depending on the method of weight loss (i.e., behavioral or surgical) (53). A relationship between BMI and activation of areas involved in reward anticipation and impulsivity [anterior cingulate cortex (49) and middle frontal gyrus (52)] was identified in two studies. 


\section{HEALTHY WEIGHT PARTICIPANTS}

The most common finding across studies of healthy weight participants was that motivational state (i.e., fasted or satiated state) affected brain activation to food. Fasting often increased responses to high-calorie foods in areas associated with processing of reward and stimuli salience $\operatorname{COFC}(66,69,71,72)$, striatum $(65,69,72)$, insula $(69,71)]$, decision making $[\operatorname{OFC}(66,69,71,72)$, striatum $(65,69,72)]$, implicit learning $[\operatorname{OFC}(66,69,71,72)$, putamen $(66,71)]$, and the processing of visual cues [fusiform gyrus (68, $71,74)]$. Gender differences were identified in responses to food cues with females displaying greater activation in a variety of brain regions implicated in attention, emotion, recollective memory, and decision making $(67,68,74)$.

\section{OBESE PARTICIPANTS}

In obese participants, food compared to non-food images activated areas including the PFC, insula, amygdala, nucleus accumbens $(91,92)$, and cerebellum (89). These areas are associated with numerous roles which could affect food cue processing including executive functioning, reward processing, and anticipation, reinforcement learning, memory modulation, and motor control. Females showed greater activation in the caudate and OFC when fasted and greater activation in the anterior cingulate cortex when satiated (88). Abdominal adiposity predicted brain activity in one study (92).

\section{RESULTS OF THE ALE META-ANALYSIS}

As only one study reported increases in brain activation following weight loss, only studies reporting decreases in neural activation from pre- to post-weight loss were included in the meta-analysis. Five studies describing seven experiments were identified that met the meta-analysis inclusion criteria with 45 participants and 41 foci (55-59). The meta-analysis identified 13 clusters, which survived statistical thresholds, as demonstrated in Figure 2. The largest cluster was the left superior temporal gyrus (MNI: $-40,-48,6$ ), as described in Table S3 in Supplementary Material. Other clusters surviving statistical thresholds included right middle frontal gyrus (MNI: 32, 34, 34), left lentiform nucleus (MNI: -12, 0, -2), left cingulate gyrus (MNI: $-4,-34,26$,), and right precentral gyrus (MNI: 40, 0, 42).

\section{DISCUSSION}

This is the first systematic review to investigate neural responses to visual food cues across all weight categories and provide a metaanalysis of neural changes before and after weight loss. This review highlights that neural responses to visual food cues differ depending on the weight status of individuals, and changes in neural activation patterns are observed pre- to post-weight loss. More specifically, obese individuals compared to healthy weight had increased activation to foods compared to non-foods, and this was most pronounced in response to energy dense foods. This was evident in brain regions associated with the anticipation of the rewarding value of the food, emotion and memory associated with previous experiences with the food, and visual processing of the food cues. Obese individuals were more responsive to food cues in the satiated state compared to healthy weight individuals. Additionally, weight loss reduced neural responses in areas related
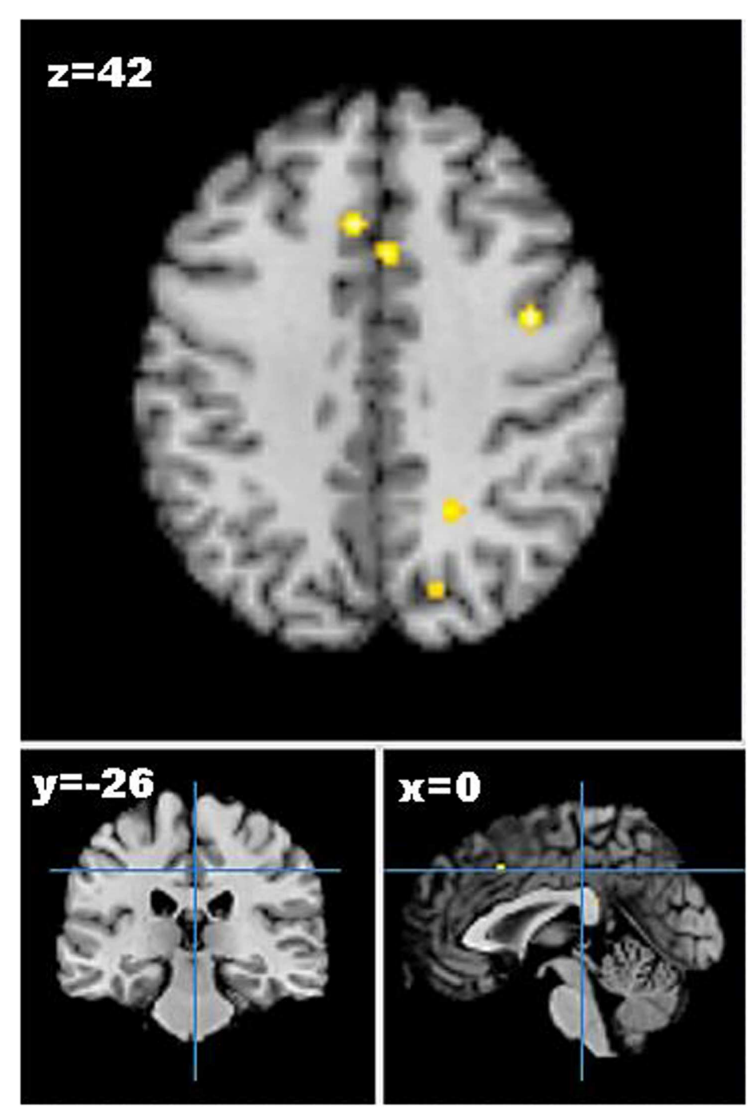

FIGURE 2 | Axial ( $z$ ), coronal $(y)$, and sagittal $(x)$ views of decreased activation in studies comparing neural activation to visual food cues from pre- to post-weight loss, as detected by fMRI. FDR corrected $P<0.05$, cluster size $>100 \mathrm{~mm}^{3}$, region of interest studies excluded. Figure shows decreased activation from pre- to post-weight loss in the cingulate gyrus, middle frontal gyrus, and precuneus.

to executive functioning, impulsivity, and reinforcement learning to visual food cues, despite differences in the modality of the intervention. However, inconsistent activation patterns were reported across studies, which may be attributable to the variety of participant groups recruited, pre-scan preparation, and the chosen fMRI parameters (e.g., block design vs. event-related). The fMRI findings of the current review are consistent with previous published reviews in the area (19-21).

When comparing healthy weight and overweight/obese participants, increased reward-related responses to food (e.g., insula and OFC), particularly high-calorie foods, compared to non-food were found in obese participants. It has been suggested that obesity may be linked to an increase in neural-related reward anticipation from food cues, and a decrease in reward during food consumption. This could potentiate overeating to compensate for imbalances in the neural reward pathways and subsequent diminished experience of reward $(7,97)$. Although only three studies reported a correlation between neural responses and BMI $(37,44,47)$, the increased responses reported in these studies of obese participants compared to normal-weight participants may explain some 
individual's vulnerability to overeating, food cues, and possible diet failures $(7,10)$. Similar findings have been reported by GarciaGarcia et al. and Asmaro et al. who noted differential activation patterns between obese and healthy weight individuals to the sight of food, particularly in reward-related regions. However, individuals with binge-eating disorder and studies using taste stimuli were also included in previous reviews, which may have recruited additional areas of the brain and potentially confound the findings of these studies.

This review extends current literature regarding neural responses to visual food cues by examining brain activation by weight status category (i.e., healthy weight, overweight/obese) as well as pre- and post-weight-loss responses. Results from weight loss studies suggest that gastric bypass surgery reduces reward responses from pre- to post-surgery (49, 52, 57-59). Although consistent reductions in neural activation were observed irrespective of method of weight loss, changes in activation via surgical weight loss differed to activation changes observed in behavioral weight loss that focused on diet and exercise (53). Interestingly, in both behavioral and surgical interventions, brain activity prior to weight loss in areas related to reward anticipation and impulsivity (e.g., anterior cingulate cortex and nucleus accumbens), and decision making (dorsolateral PFC) predicted degree of weight loss success. This provides insight that both surgical and behavioral weight loss may be underpinned by a neural mechanism as well as restriction of the amount of food consumed. In addition, individuals who had maintained successful weight loss showed increased neural activity in regions associated with inhibitory control compared to obese individuals and increased responses in areas related to memory compared to healthy weight individuals $(50,51,55,56)$. The findings of the review highlight that neural-related mechanisms may make some people more predisposed to weight regain, despite successful loss of weight. This may have important implications for obesity follow up and treatment, and provides evidence that neural mechanisms may affect weight loss success or predict proneness to relapse. The high cost of MRI precludes large scale scanning of subjects engaged in weight-loss programs, but use of fMRI in focused clinical trials could be used to validate changes in neurocircuitry patterns associated with successful maintenance of weight loss.

The results of the meta-analysis revealed that there were some small regions of convergence of brain responsivity across weightloss interventions. Deactivation was observed from pre- to postweight loss in areas involved in emotion and memory (e.g., cingulate gyrus and precuneus), visual processing (e.g., superior occipital gyrus), learning centers (e.g., lentiform nucleus and cingulate gyrus), and motor regions (e.g., precentral gyrus and lentiform nucleus) (96). This may imply that individuals who have experienced weight loss regardless of modality also have changes in neural activation associated with memory and emotion of previous experiences with the food as well as alterations in the processing of external food inputs. This also suggests that individuals who have experienced weight loss have corresponding changes in activation based on behaviors that are implicitly learned or reinforced during weight-loss interventions. Additionally, it appears that weight loss could result in changes in the planning and regulation of movements associated with eating such as reaching to obtain the food, chewing, and swallowing. Although several areas survived statistical thresholds, volume of activation was small. This suggests that while there were commonalities across studies regarding reductions in neural activity to food cues across weightloss interventions, the areas of congruence are minimal. This is likely due to the overall number of studies included and the pooling of different modalities of weight loss into a single meta-analysis and thus results should be interpreted accordingly.

Significant differences were found depending on the participant's motivational state across all weight categories, although fasting times were inconsistent across studies. Obese individuals were found to have activation consistent with continued reward processing (e.g., PFC, OFC, caudate) and emotional responses (e.g., insula, caudate, amygdala) to food cues following a meal compared to healthy weight controls. That is, obese individuals appear to be more reactive to high-calorie foods and have continued reward processing even following a meal. These findings are consistent with previous literature in this area $(21,23,24)$. This is significant as it could provide a neural mechanism for overeating in obese individuals, with neural stimulation even in the state of satiety. In the fasting state, obese individuals were found to have increased activation in areas associated with the anticipation of reward while healthy weight controls were found to have greater activation in areas associated with cognitive control. This suggests that BMI and the hunger-state will greatly affect an individual's natural desire for food and food reward responsiveness, as well as food choices and subsequent caloric intake (14). This provides preliminary data that could be used in weight interventions regarding meal timing to avoid excessive anticipation for food and subsequent overeating. In addition, notable gender bias was found in the current review, with females displaying differential activation patterns compared to males in regions associated with reward anticipation, food motivation, and inhibitory control based on motivational state, i.e., fasted or satiated. $(67,68,74,88)$. This may indicate that gender influences an individual's susceptibility to addictive-like eating behaviors.

The term 'food addiction' has emerged and is being used increasingly in lay literature $(98,99)$ in association with specific eating patterns and rising levels of obesity $(22,30,77,96,100)$. It has been postulated that food addiction is associated with specific food-related behaviors including: tolerance to large amounts of food, persistent desire, or craving for specific foods and lack of control over the amount of food consumed (77). In this way food addiction would share similar clinical characteristics that overlap with drug dependence and other common types of addiction, as defined by the Diagnostic and Statistical manual of Mental Disorders version 5 (DSM-V) $(29,77,101-103)$. Although, there is debate regarding to the inclusion of food addiction as a DSM-V eating and feeding disorder (3), currently no universally accepted definition for food addiction exists. Findings from this review provide preliminary support for the food addiction hypothesis. Areas of the reward network including the mesolimbic and nigrostriatal regions, which have been implicated in other addictions including drug and alcohol abuse, were activated in response to visual food cues. However, there is a great deal of variation in the brain areas activated across reviewed studies, as well as the experimental conditions used (e.g., motivational state, images presented, fMRI 
parameters). This indicates that further research utilizing a standardized approach is required to substantiate study findings to either support or refute the existence of food addiction as a distinct phenomenon. This is in line with the findings of Ziauddeen et al. (19) who concluded that while neural activation in healthy weight individuals is somewhat consistent across studies, activation in obese, and binge eaters is too varied to categorically confirm the existence of food addiction.

Although little evidence exists to confirm the types of food associated with food addiction, it is commonly assumed that "junk foods" high in calories, sugar, and fat such as chocolate and potato chips are typical foci of addictive-like tendencies such as craving $(27,76,104)$. The range of classifications given to food images in this review such as "high" and "low" calorie foods and "palatable" and "bland" foods appears subjective and arbitrary, with no studies consulting a dietitian or nutritionist. Only seven studies specifically identified actual calorific values of foods and the majority of studies did not pilot test images or provide rationale for their selection of presented foods. The selection of foods in research studies is important to ascertain if it is a universal chemical component of food (e.g., selection based sugar or fat content), a whole category of food (e.g., selection of 'junk food'), or more simply a personal preference (e.g., selection by administering a pre-scan survey) that could make a food more likely to be overeaten. This could provide further insight into whether food addiction is a plausible phenomenon. Furthermore, the range of control images presented were variable and included cars, landscapes, and blurred images. As these images were not standardized across studies, it is unknown as to whether these assorted images may elicit different neural patterns from one another based on their perceived valence and arousal. The variability of food images used in studies has been previously acknowledged and a database of standardized pictures based on image characteristics and nutrient composition has recently been developed (105). Future studies should consider the use of such a database to facilitate the standardization of images and comparability across studies.

The design of an experimental paradigm for fMRI studies requires extensive planning including behavioral predictions of cognitive tasks and the formulation of a hypothesis to inform the task conditions and image acquisition parameters. Ideally, fMRI experiments cover the whole brain with the highest spatial resolution achievable in the shortest time. The trans-axial plane is usually chosen to cover the whole brain in as few slices as possible. In some cases, the acquisition plane is angled to avoid regions of high magnetic susceptibility (e.g., air-tissue and bone-tissue interfaces) that can adversely affect image quality, such as in targeting the reward network. The block design has dominated fMRI due to its ease of implementation, robustness of results, increased statistical power, and relatively large BOLD signal relative to baseline (106, 107). However, blocked paradigms have poorer temporal resolution and are susceptible to stimulus correlated movement artifacts. Event-related paradigms measure responses to single events (typically over $0.5 \mathrm{~s}$ ), and then combine a large number of those events to improve statistical power. This is advantageous to detect transient variations in hemodynamic responses, reduce the subject's ability to predict the next event, and allows for post hoc sorting of trials and correlations with other variables (106, 107). Generally, blocked paradigms are most useful to localize activation in brain regions associated with a particular task while event-related paradigms allow for a more in depth investigation of the response profile in an identified brain region.

Across the reviewed studies, fMRI results were not reported in a consistent method. Talairach or MNI coordinates, used to describe the location of brain structures independent from individual differences in the size and overall shape of the brain, were reported in the majority of studies. Using the standardized coordinates allows the comparison of brain region activation with other studies, increasing the power of results when combined and can provide a method for meta-analysis (25). Cluster size or volume indicates that the area of neural activation reported in the study is large enough to be statistically plausible rather than just an error in measurement, improving the quality of the study (108). Only half of the studies included in the review reported cluster size or volume.

This review is limited by the heterogeneity of study variables used across the reviewed articles, making direct comparisons between studies difficult. As a limited number of studies met the inclusion criteria for the meta-analysis comparing pre- and postweight loss activation changes, a single exploratory meta-analysis was undertaken combining both surgical and behavioral weightloss interventions. The pooling of different methods of weight loss could impact the findings of the meta-analysis. However, this data provides insight into neural activation following weight loss, regardless of modality, which needs to be substantiated in future research when more studies have been published in this area. The quality of reporting BMI was inconsistent across studies, with participant groups containing BMI ranges that corresponded with more than one of the WHO categories (54). This could affect possible relationships between $\mathrm{fMRI}$ outcomes and weight status. As BMI can be affected by numerous factors including muscle mass, adiposity or body fat percentage is a more reflective measure of obesity assessment and should be considered for use in future studies. The majority of reviewed articles studied adult female participants exclusively, potentially limiting the generalizability of the study findings to other population groups. Finally, the broad age range of the study participants could be a potential limitation of the review, with previous studies showing age-related changes in neural activity including reduced sensitivity of brain areas associated with satiety (109).

Strengths of this review include the standardization of populations studied and stimuli used to visual food cues exclusively. The use of different stimuli modalities including food consumption (104, 110-122), odors (123-125), and intravenous infusion (126) recruits additional areas of the brain including taste, texture, olfactory, and food intake centers, potentially confounding results. Only visual food cues have been included in the current review in order to minimize the activation of additional areas of the brain. Additionally, the exclusion of different populations, including children and adolescents $(127,128)$, eating disorders including binge-eating disorder, anorexia nervosa and bulimia nervosa (129$134)$, and neurological disorders $(39,135)$, reduces the chance of additional confounding factors contributing to the variations in neural responses to visual food cues. This exclusion criterion is consistent with previous literature in the field $(28,38,43)$. 
It is recommended that future studies report detailed nutritional information of images presented during scanning and BMI classification using the WHO guidelines. This field of research requires the involvement of a multidisciplinary team including imaging specialists, neuroscientists, psychologists, and dietitians to ensure high quality study design. Further, studies should use validated eating behavior questionnaires and use within participant cross-over study design to investigate the impact of motivational state on neural responses. Weight-loss studies using fMRI as an outcome measure should routinely report brain coordinates, cluster size and threshold. Future meta-analyses in this area should investigate responsivity to food cues by specific mode of weight loss (i.e., surgical or behavioral) as these are likely to elicit different changes in neural responses.

\section{CONCLUSION}

This review found that neural activation differed based on weight status with obese individuals displaying increased responses to food compared to non-food and continued responsivity to food following a meal. This suggests that neural activity to food cues could be an additional mechanism contributing to the pathogenesis of overeating and subsequent weight gain. Regions of activation differed across the reviewed studies due to a wide range of study conditions used and inconsistency in reporting of findings. The meta-analysis undertaken revealed changes in brain activation patterns following weight loss. However, the small cluster sizes suggest that there is minimal congruence of neural activation across weight-loss studies. Future fMRI studies examining neural activation in response to visual food cues should standardize reporting of nutrition variables and fMRI outcomes to allow for more direct comparisons between studies.

\section{ACKNOWLEDGMENTS}

The authors would like to thank Dr Melinda Hutchesson for her assistance in manuscript preparation. Kirrilly M. Pursey is supported by the Hunter Valley Research Foundation's Robin McDonald Regional Research Memorial scholarship.

\section{SUPPLEMENTARY MATERIAL}

The Supplementary Material for this article can be found online at http://journal.frontiersin.org/Journal/10.3389/fnut.2014.00007/ abstract

\section{REFERENCES}

1. Ng M, Fleming T, Robinson M, Thomson B, Graetz N, Margono C, et al. Global, regional, and national prevalence of overweight and obesity in children and adults during 1980-2013: a systematic analysis for the Global Burden of Disease Study 2013. Lancet (2014). doi:10.1016/S0140-6736(14)60460-8

2. Ogden CL, Carroll MD, Kit BK, Flegal KM. Prevalence of childhood and adult obesity in the United States, 2011-2012. J Am Med Assoc (2014) 311(8):806-14. doi:10.1001/jama.2014.732

3. World Health Organization. World Health Statistics: Global Health Statistics. Geneva: World Health Organization (2014).

4. Hughes A, Farewell K, Harris D, Reilly J. Quality of life in a clinical sample of obese children. Int J Obes (2007) 31:39-44. doi:10.1038/sj.ijo.0803410

5. Colquitt JL, Picot J, Loveman E, Clegg AJ. Surgery for obesity. Cochrane Database Syst Rev (2009) 2:CD003641. doi:10.1002/14651858.CD003641.pub3

6. Appel LJ, Clark JM, Yeh HC, Wang NY, Coughlin JW, Daumit G, et al. Comparative effectiveness of weight-loss interventions in clinical practice. New Engl J Med (2011) 365(21):1959-68. doi:10.1056/NEJMoa1108660
7. Burger KS, Stice E. Variability in reward responsivity and obesity: evidence from brain imaging studies. Curr Drug Abuse Rev (2011) 4(3):182-9. doi:10.2174/1874473711104030182

8. Koob GF, Volkow ND. Neurocircuitry of addiction. Neuropsychopharmacology (2010) 35(1):217-38. doi:10.1038/npp.2009.110

9. Carnell S, Gibson C, Benson L, Ochner CN, Geliebter A. Neuroimaging and obesity: current knowledge and future directions. Obes Rev (2012) 13(1):43-56. doi:10.1111/j.1467-789X.2011.00927.x

10. Dagher A. Functional brain imaging of appetite. Trends Endocrinol Metab (2012) 23(5):250-60. doi:10.1016/j.tem.2012.02.009

11. Stice E, Spoor S, Bohon C, Small DM. Relation between obesity and blunted striatal response to food is moderated by TaqIA Al allele. Science (2008) 322(5900):449-52. doi:10.1126/science.1161550

12. Stice E, Yokum S, Bohon C, Marti N, Smolen A. Reward circuitry responsivity to food predicts future increases in body mass: moderating effects of DRD2 and DRD4. Neuroimage (2010) 50(4):1618-25. doi:10.1016/j.neuroimage. 2010.01.081

13. Burger KS, Stice E. Relation of dietary restraint scores to activation of rewardrelated brain regions in response to food intake, anticipated intake, and food pictures. Neuroimage (2011) 55(1):233-9. doi:10.1016/j.neuroimage. 2010.12.009

14. García-García I, Jurado MA, Garolera M, Segura B, Sala-Llonch R, Marqués-Iturria I, et al. Alterations of the salience network in obesity: a restingstate fMRI study. Hum Brain Mapp (2012) 34(11):2786-97. doi:10.1002/hbm. 22104

15. Gearhardt AN, White MA, Masheb RM, Morgan PT, Crosby RD, Grilo CM. An examination of the food addiction construct in obese patients with binge eating disorder. Int J Eat Disord (2012) 45(5):657-63. doi:10.1002/eat.20957

16. Lemmens VEPP, Oenema A, Klepp KI, Henriksen HB, Brug J. A systematic review of the evidence regarding efficacy of obesity prevention interventions among adults. Obes Rev (2008) 9(5):446-55. doi:10.1111/j.1467-789X.2008. 00468.x

17. Brown T, Avenell A, Edmunds LD, Moore H, Whittaker V, Avery L, et al. Systematic review of long-term lifestyle interventions to prevent weight gain and morbidity in adults. Obes Rev (2009) 10(6):627-38. doi:10.1111/j.1467-789X. 2009.00641.x

18. Wilks DC, Besson H, Lindroos AK, Ekelund U. Objectively measured physical activity and obesity prevention in children, adolescents and adults: a systematic review of prospective studies. Obes Rev (2011) 12(5):e119-29. doi:10.1111/j.1467-789X.2010.00775.x

19. Ziauddeen $\mathrm{H}$, Fletcher PC. Is food addiction a valid and useful concept? Obes $\operatorname{Rev}(2013)$ 14(1):19-28. doi:10.1111/j.1467-789X.2012.01046.x

20. Garcia-Garcia I, Narberhaus A, Marques-Iturria I, Garolera M, Radoi A, Segura B, et al. Neural responses to visual food cues: insights from functional magnetic resonance imaging. Eur Eat Disord Rev (2013) 21(2):89-98. doi:10.1002/erv.2216

21. Asmaro D, Liotti M. High-caloric and chocolate stimuli processing in healthy humans: an integration of functional imaging and electrophysiological findings. Nutrients (2014) 6(1):319-41. doi:10.3390/nu6010319

22. van der Laan LN, de Ridder DTD, Viergever MA, Smeets PAM. The first taste is always with the eyes: a meta-analysis on the neural correlates of processing visual food cues. Neuroimage (2011) 55(1):296-303. doi:10.1016/j.neuroimage. 2010.11.055

23. Kennedy J, Dimitropoulos A. Influence of feeding state on neurofunctional differences between individuals who are obese and normal weight: a meta-analysis of neuroimaging studies. Appetite (2014) 75:103-9. doi:10.1016/j.appet.2013. 12.017

24. Brooks SJ, Cedernaes J, Schioth HB. Increased prefrontal and parahippocampal activation with reduced dorsolateral prefrontal and insular cortex activation to food images in obesity: a meta-analysis of fMRI studies. PLoS One (2013) 8(4):e60393. doi:10.1371/journal.pone.0060393

25. Tang DW, Fellows LK, Small DM, Dagher A. Food and drug cues activate similar brain regions: a meta-analysis of functional MRI studies. Physiol Behav (2012) 106(3):317-24. doi:10.1016/j.physbeh.2012.03.009

26. Scharmuller W, Ubel S, Ebner F, Schienle A. Appetite regulation during food cue exposure: a comparison of normal-weight and obese women. Neurosci Lett (2012) 518(2):106-10. doi:10.1016/j.neulet.2012.04.063

27. Nummenmaa L, Hirvonen J, Hannukainen JC, Immonen H, Lindroos MM, Salminen $\mathrm{P}$, et al. Dorsal striatum and its limbic connectivity mediate abnormal 
anticipatory reward processing in obesity. PLoS One (2012) 7(2):e31089. doi:10.1371/journal.pone.0031089

28. Stoeckel LE, Weller RE, Cook EW III, Twieg DB, Knowlton RC, Cox JE. Widespread reward-system activation in obese women in response to pictures of high-calorie foods. Neuroimage (2008) 41(2):636-47. doi:10.1016/j. neuroimage.2008.02.031

29. Gearhardt AN, Yokum S, Orr PT, Stice E, Corbin WR, Brownell KD. Neural correlates of food addiction. Arch Gen Psychiatry (2011) 68(8):808-16. doi:10. 1001/archgenpsychiatry.2011.32

30. Evidence Analysis Manual. Steps in the ADA Evidence Analysis Process. Chicago: American Dietetic Association (2012).

31. World Health Organization. BMI Classification. World Health Organization (2013). Available from: http://apps.who.int/bmi/index.jsp?introPage=intro_3. html

32. Eickhoff SB, Laird AR, Grefkes C, Wang LE, Zilles K, Fox PT. Coordinatebased activation likelihood estimation meta-analysis of neuroimaging data: a random-effects approach based on empirical estimates of spatial uncertainty. Hum Brain Mapp (2009) 30(9):2907-26. doi:10.1002/hbm.20718

33. Connolly L, Coveleskie K, Kilpatrick LA, Labus JS, Ebrat B, Stains J, et al. Differences in brain responses between lean and obese women to a sweetened drink. Neurogastroenterol Motil (2013) 25(7):579-e460. doi:10.1111/nmo.12125

34. Cornier MA, McFadden KL, Thomas EA, Bechtell JL, Eichman LS, Bessesen $\mathrm{DH}$, et al. Differences in the neuronal response to food in obesity-resistant as compared to obesity-prone individuals. Physiol Behav (2013) 110:122-8. doi:10.1016/j.physbeh.2013.01.002

35. Ho A, Kennedy J, Dimitropoulos A. Neural correlates to food-related behavior in normal-weight and overweight/obese participants. PLoS One (2012) 7(9):e45403. doi:10.1371/journal.pone.0045403

36. Lee Y, Chong MFF, Liu JCJ, Libedinsky C, Gooley JJ, Chen SQ, et al. Dietary disinhibition modulates neural valuation of food in the fed and fasted states. Am J Clin Nutr (2013) 97(5):919-25. doi:10.3945/ajcn.112.053801

37. Martens MJI, Born JM, Lemmens SGT, Karhunen L, Heinecke A, Goebel $\mathrm{R}$, et al. Increased sensitivity to food cues in the fasted state and decreased inhibitory control in the satiated state in the overweight. Am J Clin Nutr (2013) 97(3):471-9. doi:10.3945/ajcn.112.044024

38. Dimitropoulos A, Tkach J, Ho A, Kennedy J. Greater corticolimbic activation to high-calorie food cues after eating in obese vs. normal-weight adults. Appetite (2012) 58(1):303-12. doi:10.1016/j.appet.2011.10.014

39. Holsen LM, Savage CR, Martin LE, Bruce AS, Lepping RJ, Ko E, et al. Importance of reward and prefrontal circuitry in hunger and satiety: PraderWilli syndrome vs simple obesity. Int J Obes (Lond) (2012) 36(5):638-47. doi:10.1038/ijo.2011.204

40. Garcia-Garcia I, Jurado MA, Garolera M, Segura B, Marques-Iturria I, Pueyo R, et al. Functional connectivity in obesity during reward processing. Neuroimage (2013) 66:232-9. doi:10.1016/j.neuroimage.2012.10.035

41. Jastreboff AM, Sinha R, Lacadie C, Small DM, Sherwin RS, Potenza MN. Neural correlates of stress- and food cue-induced food craving in obesity: association with insulin levels. Diabetes Care (2013) 36(2):394-402. doi: $10.2337 / \mathrm{dc} 12-1112$

42. Kullmann S, Pape AA, Heni M, Ketterer C, Schick F, Haring HU, et al. Functional network connectivity underlying food processing: disturbed salience and visual processing in overweight and obese adults. Cereb Cortex (2013) 23(5):1247-56. doi:10.1093/cercor/bhs124

43. Frankort A, Roefs A, Siep N, Roebroeck A, Havermans R, Jansen A. Reward activity in satiated overweight women is decreased during unbiased viewing but increased when imagining taste: an event-related fMRI study. Int J Obes (Lond) (2012) 36(5):627-37. doi:10.1038/ijo.2011.213

44. Grosshans M, Vollmert C, Vollstadt-Klein S, Tost H, Leber S, Bach P, et al. Association of leptin with food cue-induced activation in human reward pathways. Arch Gen Psychiatry (2012) 69(5):529-37. doi:10.1001/archgenpsychiatry. 2011.1586

45. Ng J, Stice E, Yokum S, Bohon C. An fMRI study of obesity, food reward, and perceived caloric density. Does a low-fat label make food less appealing? Appetite (2011) 57(1):65-72. doi:10.1016/j.appet.2011.03.017

46. Stoeckel LE, Kim J, Weller RE, Cox JE, Cook EW III, Horwitz B. Effective connectivity of a reward network in obese women. Brain Res Bull (2009) 79(6):388-95. doi:10.1016/j.brainresbull.2009.05.016
47. Rothemund Y, Preuschhof C, Bohner G, Bauknecht H-C, Klingebiel R, Flor $\mathrm{H}$, et al. Differential activation of the dorsal striatum by high-calorie visual food stimuli in obese individuals. Neuroimage (2007) 37(2):410-21. doi:10.1016/j.neuroimage.2007.05.008

48. Frank S, Wilms B, Veit R, Ernst B, Thurnheer M, Kullmann S, et al. Altered brain activity in severely obese women may recover after Roux-en Y gastric bypass surgery. Int J Obes (Lond) (2014) 38(3):341-8. doi:10.1038/ijo.2013.60

49. Goldman RL, Canterberry M, Borckardt JJ, Madan A, Byrne TK, George MS, et al. Executive control circuitry differentiates degree of success in weight loss following gastric-bypass surgery. Obesity (Silver Spring) (2013) 21(11):2189-96. doi:10.1002/oby.20575

50. McCaffery JM, Haley AP, Sweet LH, Phelan S, Raynor HA, Del Parigi A, et al. Differential functional magnetic resonance imaging response to food pictures in successful weight-loss maintainers relative to normal-weight and obese controls. Am J Clin Nutr (2009) 90(4):928-34. doi:10.3945/ajcn.2009.27924

51. Tregellas JR, Wylie KP, Rojas DC, Tanabe J, Martin J, Kronberg E, et al. Altered default network activity in obesity. Obesity (Silver Spring) (2011) 19(12):2316-21. doi:10.1038/oby.2011.119

52. Bruce JM, Hancock L, Bruce A, Lepping RJ, Martin L, Lundgren JD, et al. Changes in brain activation to food pictures after adjustable gastric banding. Surg Obes Rel Dis (2011) 8(5):602-8. doi:10.1016/j.soard.2011.07.006

53. Bruce AS, Bruce JM, Ness AR, Lepping RJ, Malley S, Hancock L, et al. A comparison of functional brain changes associated with surgical versus behavioral weight loss. Obesity (Silver Spring) (2014) 22(2):337-43. doi:10.1002/oby. 20630

54. Weygandt M, Mai K, Dommes E, Leupelt V, Hackmack K, Kahnt T, et al. The role of neural impulse control mechanisms for dietary success in obesity. Neuroimage (2013) 83:669-78. doi:10.1016/j.neuroimage.2013.07.028

55. Murdaugh DL, Cox JE, Cook EW III, Weller RE. fMRI reactivity to high-calorie food pictures predicts short- and long-term outcome in a weight-loss program. Neuroimage (2012) 59(3):2709-21. doi:10.1016/j.neuroimage.2011.10.071

56. Nock NL, Dimitropolous A, Tkach J, Frasure H, von Gruenigen V. Reduction in neural activation to high-calorie food cues in obese endometrial cancer survivors after a behavioral lifestyle intervention: a pilot study. BMC Neurosci (2012) 13(1):74. doi:10.1186/1471-2202-13-74

57. Ochner CN, Laferrere B, Afifi L, Atalayer D, Geliebter A, Teixeira J. Neural responsivity to food cues in fasted and fed states pre and post gastric bypass surgery. Neurosci Res (2012) 74(2):138-43. doi:10.1016/j.neures.2012.08.002

58. Ochner CN, Stice E, Hutchins E, Afifi L, Geliebter A, Hirsch J, et al. Relation between changes in neural responsivity and reductions in desire to eat highcalorie foods following gastric bypass surgery. Neuroscience (2012) 209:128-35. doi:10.1016/j.neuroscience.2012.02.030

59. Ochner CN, Kwok Y, Conceição E, Pantazatos SP, Puma LM, Carnell S, et al. Selective reduction in neural responses to high calorie foods following gastric bypass surgery. Ann Surg (2011) 253(3):502-7. doi:10.1097/SLA. 0b013e318203a289

60. Benedict C, Brooks SJ, O’Daly OG, Almèn MS, Morell A, Åberg K, et al. Acute sleep deprivation enhances the brain's response to hedonic food stimuli: an fMRI study. JClin Endocrinol Metab (2012) 97(3):E443-7. doi:10.1210/jc.20112759

61. Karra E, O’Daly OG, Choudhury AI, Yousseif A, Millership S, Neary MT, et al. A link between FTO, ghrelin, and impaired brain food-cue responsivity. J Clin Invest (2013) 123(8):3539-51. doi:10.1172/JCI44403

62. Kroemer NB, Krebs L, Kobiella A, Grimm O, Pilhatsch M, Bidlingmaier M, et al. Fasting levels of ghrelin covary with the brain response to food pictures. Addict Biol (2013) 18(5):855-62. doi:10.1111/j.1369-1600.2012.00489.x

63. Kroemer NB, Krebs L, Kobiella A, Grimm O, Vollstadt-Klein S, Wolfensteller $\mathrm{U}$, et al. (Still) longing for food: insulin reactivity modulates response to food pictures. Hum Brain Mapp (2013) 34(10):2367-80. doi:10.1002/hbm. 22071

64. Evero N, Hackett LC, Clark RD, Phelan S, Hagobian TA. Aerobic exercise reduces neuronal responses in food reward brain regions. J Appl Physiol (2012) 112(9):1612-9. doi:10.1152/japplphysiol.01365.2011

65. Born JM, Lemmens SGT, Martens MJI, Formisano E, Goebel R, WesterterpPlantenga MS. Differences between liking and wanting signals in the human brain and relations with cognitive dietary restraint and body mass index. Am J Clin Nutr (2011) 94(2):392-403. doi:10.3945/ajcn.111.012161 
66. Born JM, Lemmens SGT, Rutters F, Nieuwenhuizen AG, Formisano E, Goebel $\mathrm{R}$, et al. Acute stress and food-related reward activation in the brain during food choice during eating in the absence of hunger. Int J Obes (Lond) (2010) 34(1):172-81. doi:10.1038/ijo.2009.221

67. Cornier M-A, Salzberg AK, Endly DC, Bessesen DH, Tregellas JR. Sex-based differences in the behavioral and neuronal responses to food. Physiol Behav (2010) 99(4):538-43. doi:10.1016/j.physbeh.2010.01.008

68. Frank S, Laharnar N, Kullmann S, Veit R, Canova C, Hegner YL, et al. Processing of food pictures: influence of hunger, gender and calorie content. Brain Res (2010) 1350:159-66. doi:10.1016/j.brainres.2010.04.030

69. Goldstone AP, Prechtl De Hernandez CG, Beaver JD, Muhammed K, Croese C, Bell G, et al. Fasting biases brain reward systems towards high-calorie foods. Eur J Neurosci (2009) 30(8):1625-35. doi:10.1111/j.1460-9568.2009.06949.x

70. Coletta M, Platek S, Mohamed FB, van Steenburgh JJ, Green D, Lowe MR. Brain activation in restrained and unrestrained eaters: an fMRI study. JAbnorm Psychol (2009) 118(3):598-609. doi:10.1037/a0016201

71. Siep N, Roefs A, Roebroeck A, Havermans R, Bonte ML, Jansen A. Hunger is the best spice: an fMRI study of the effects of attention, hunger and calorie content on food reward processing in the amygdala and orbitofrontal cortex. Behav Brain Res (2009) 198(1):149-58. doi:10.1016/j.bbr.2008.10.035

72. Fuhrer D, Zysset S, Stumvoll M. Brain activity in hunger and satiety: an exploratory visually stimulated FMRI study. Obesity (Silver Spring) (2008) 16(5):945-50. doi:10.1038/oby.2008.33

73. Cornier MA, Von Kaenel SS, Bessesen DH, Tregellas JR. Effects of overfeeding on the neuronal response to visual food cues. Am J Clin Nutr (2007) 86(4):965-71.

74. Uher R, Treasure J, Heining M, Brammer MJ, Campbell IC. Cerebral processing of food-related stimuli: effects of fasting and gender. Behav Brain Res (2006) 169(1):111-9. doi:10.1016/j.bbr.2005.12.008

75. Demos KE, Kelley WM, Heatherton TF. Dietary restraint violations influence reward responses in nucleus accumbens and amygdala. J Cogn Neurosci (2011) 23(8):1952-63. doi:10.1162/jocn.2010.21568

76. Rolls ET, McCabe C. Enhanced affective brain representations of chocolate in cravers vs. non-cravers. Eur J Neurosci (2007) 26(4):1067-76. doi:10.1111/j. 1460-9568.2007.05724.x

77. Grabenhorst F, Schulte FP, Maderwald S, Brand M. Food labels promote healthy choices by a decision bias in the amygdala. Neuroimage (2013) 74:152-63. doi:10.1016/j.neuroimage.2013.02.012

78. Hollmann M, Hellrung L, Pleger B, Schlogl H, Kabisch S, Stumvoll M, et al. Neural correlates of the volitional regulation of the desire for food. Int J Obes (Lond) (2012) 36(5):648-55. doi:10.1038/ijo.2011.125

79. Lawrence NS, Hinton EC, Parkinson JA, Lawrence AD. Nucleus accumbens response to food cues predicts subsequent snack consumption in women and increased body mass index in those with reduced self-control. Neuroimage (2012) 63(1):415-22. doi:10.1016/j.neuroimage.2012.06.070

80. Siep N, Roefs A, Roebroeck A, Havermans R, Bonte M, Jansen A. Fighting food temptations: the modulating effects of short-term cognitive reappraisal, suppression and up-regulation on mesocorticolimbic activity related to appetitive motivation. Neuroimage (2012) 60(1):213-20. doi:10.1016/j.neuroimage.2011. 12.067

81. Schur EA, Kleinhans NM, Goldberg J, Buchwald D, Schwartz MW, Maravilla $\mathrm{K}$. Activation in brain energy regulation and reward centers by food cues varies with choice of visual stimulus. Int J Obes (Lond) (2009) 33(6):653-61. doi:10.1038/ijo.2009.56

82. Passamonti L, Rowe JB, Schwarzbauer C, Ewbank MP, von dem Hagen E, Calder AJ. Personality predicts the brain's response to viewing appetizing foods: the neural basis of a risk factor for overeating. J Neurosci (2009) 29(1):43-51. doi:10.1523/JNEUROSCI.4966-08.2009

83. Killgore WDS, Young AD, Femia LA, Bogorodzki P, Rogowska J, YurgelunTodd DA. Cortical and limbic activation during viewing of high- versus low-calorie foods. Neuroimage (2003) 19(4):1381-94. doi:10.1016/S10538119(03)00191-5

84. Killgore WDS, Yurgelun-Todd DA. Body mass predicts orbitofrontal activity during visual presentations of high-calorie foods. Neuroreport (2005) 16(8):859-63. doi:10.1097/00001756-200505310-00016

85. Killgore WDS, Yurgelun-Todd DA. Affect modulates appetite-related brain activity to images of food. Int J Eat Disord (2006) 39(5):357-63. doi:10.1002/ eat. 20240
86. Porubska K, Veit R, Preissl H, Fritsche A, Birbaumer N. Subjective feeling of appetite modulates brain activity. An fMRI study. Neuroimage (2006) 32(3):1273-80. doi:10.1016/j.neuroimage.2006.04.216

87. Simmons WK, Martin A, Barsalou LW. Pictures of appetizing foods activate gustatory cortices for taste and reward. Cereb Cortex (2005) 15(10):1602-8. doi:10.1093/cercor/bhi038

88. Geliebter A, Pantazatos SP, McOuatt H, Puma L, Gibson CD, Atalayer D. Sexbased fMRI differences in obese humans in response to high vs. low energy food cues. Behav Brain Res (2013) 243:91-6. doi:10.1016/j.bbr.2012.12.023

89. Lundgren JD, Patrician TM, Breslin FJ, Martin LE, Donnelly JE, Savage CR. Evening hyperphagia and food motivation: a preliminary study of neural mechanisms. Eat Behav (2013) 14(4):447-50. doi:10.1016/j.eatbeh. 2013.08.006

90. Tryon MS, Carter CS, DeCant R, Laugero KD. Chronic stress exposure may affect the brain's response to high calorie food cues and predispose to obesogenic eating habits. Physiol Behav (2013) 120:233-42. doi:10.1016/j.physbeh. 2013.08.010

91. Van Vugt DA, Krzemien A, Alsaadi H, Palerme S, Reid RL. Effect of insulin sensitivity on corticolimbic responses to food picture in women with polycystic ovary syndrome. Obesity (Silver Spring) (2013) 21(6):1215-22. doi:10.1002/ oby. 20148

92. Luo S, Romero A, Adam TC, Hu HH, Monterosso J, Page KA. Abdominal fat is associated with a greater brain reward response to high-calorie food cues in hispanic women. Obesity (Silver Spring) (2013) 21(10):2029-36. doi:10.1002/oby.20344

93. Beaver JD, Lawrence AD, van Ditzhuijzen J, Davis MH, Woods A, Calder AJ. Individual differences in reward drive predict neural responses to images of food. J Neurosci (2006) 26(19):5160-6. doi:10.1523/JNEUROSCI.0350-06. 2006

94. St-Onge MP, Sy M, Heymsfield SB, Hirsch J. Human cortical specialization for food: a functional magnetic resonance imaging investigation. J Nutr (2005) 135(5):1014-8.

95. LaBar KS, Gitelman DR, Parrish TB, Kim Y-H, Nobre AC, Mesulam MM. Hunger selectively modulates corticolimbic activation to food stimuli in humans. Behav Neurosci (2001) 115(2):493-500. doi:10.1037/0735-7044.115. 2.493

96. Kandel E, Schwartz J, Jessell T, Siegelbaum S, Hudspeth A. Principles of Neural Science. 5th ed. New York: McGraw-Hill (2012).

97. Volkow ND, Wang G-J, Baler RD. Reward, dopamine and the control of food intake: implications for obesity. Trends Cogn Sci (2011) 15(1):37-46. doi:10.1016/j.tics.2010.11.001

98. Reilly R. Food Addiction DOES Exist: Sugar-Laden Junk Activates the Same Region of the Brain Affected by Heroin and Cocaine (2013). Available from: http://www.dailymail.co.uk/health/article-2402746/Food-addiction-DOESexist-Junk-food-activates-region-brain-hard-drugs.html\#ixzz2fIIAU9jZ

99. Food Addicts Anonymous (2013). Available from: http://www.foodaddicts anonymous.org/

100. Clark SM, Saules KK. Validation of the Yale food addiction scale among a weight-loss surgery population. Eat Behav (2013) 14(2):216-9. doi:10.1016/j. eatbeh.2013.01.002

101. Zhang Y, von Deneen KM, Tian J, Gold MS, Liu Y. Food addiction and neuroimaging. Curr Pharm Des (2011) 17(12):1149-57. doi:10.2174/ 138161211795656855

102. Liu Y, Von Deneen KM, Kobeissy FH, Gold MS. Food addiction and obesity: evidence from bench to bedside. J Psychoactive Drugs (2010) 42(2):133-45. doi:10.1080/02791072.2010.10400686

103. Ifland JR, Preuss HG, Marcus MT, Rourk KM, Taylor WC, Burau K, et al. Refined food addiction: a classic substance use disorder. Med Hypotheses (2009) 72(5):518-26. doi:10.1016/j.mehy.2008.11.035

104. Smeets PAM, De Graaf C, Stafleu A, Van Osch MJP, Nievelstein RAJ, Van Der Grond J. Effect of satiety on brain activation during chocolate tasting in men and women. Am J Clin Nutr (2006) 83(6):1297-305.

105. Blechert J, Meule A, Busch NA, Ohla K. Food-pics: an image database for experimental research on eating and appetite. Front Psychol (2014) 5:617. doi:10.3389/fpsyg.2014.00617

106. Chee MWL, Venkatraman V, Westphal C, Siong SC. Comparison of block and event-related fMRI designs in evaluating the word-frequency effect. Hum Brain Mapp (2003) 18(3):186-93. doi:10.1002/hbm.10092 
107. Amaro E Jr., Barker GJ. Study design in fMRI: basic principles. Brain Cogn (2006) 60(3):220-32. doi:10.1016/j.bandc.2005.11.009

108. Hayasaka S, Nichols TE. Validating cluster size inference: random field and permutation methods. Neuroimage (2003) 20(4):2343-56. doi:10.1016/ j.neuroimage.2003.08.003

109. Cheah YS, Lee S, Ashoor G, Nathan Y, Reed LJ, Zelaya FO, et al. Ageing diminishes the modulation of human brain responses to visual food cues by meal ingestion. Int J Obes (Lond) (2013). doi:10.1038/ijo.2013.237

110. Berns GS, McClure SM, Pagnoni G, Montague PR. Predictability modulates human brain response to reward. J Neurosci (2001) 21(8):2793-8.

111. Green E, Jacobson A, Haase L, Murphy C. Reduced nucleus accumbens and caudate nucleus activation to a pleasant taste is associated with obesity in older adults. Brain Res (2011) 1386:109-17. doi:10.1016/j.brainres.2011.02.071

112. Stice E, Yokum S, Blum K, Bohon C. Weight gain is associated with reduced striatal response to palatable food. J Neurosci (2010) 30(39):13105-9. doi:10. 1523/JNEUROSCI.2105-10.2010

113. De Araujo IE, Rolls ET. Representation in the human brain of food texture and oral fat. J Neurosci (2004) 24(12):3086-93. doi:10.1523/JNEUROSCI.0130-04. 2004

114. Kringelbach ML, O'Doherty J, Rolls ET, Andrews C. Activation of the human orbitofrontal cortex to a liquid food stimulus is correlated with its subjective pleasantness. Cereb Cortex (2003) 13(10):1064-71. doi:10.1093/cercor/13.10. 1064

115. Smeets PA, de Graaf C, Stafleu A, van Osch MJ, van der Grond J. Functional MRI of human hypothalamic responses following glucose ingestion. Neuroimage (2005) 24(2):363-8. doi:10.1016/j.neuroimage.2004.07.073

116. Smeets PA, de Graaf C, Stafleu A, van Osch MJ, van der Grond J. Functional magnetic resonance imaging of human hypothalamic responses to sweet taste and calories. Am J Clin Nutr (2005) 82(5):1011-6.

117. Haase L, Green E, Murphy C. Males and females show differential brain activation to taste when hungry and sated in gustatory and reward areas. Appetite (2011) 57(2):421-34. doi:10.1016/j.appet.2011.06.009

118. Frank GKW, Oberndorfer TA, Simmons AN, Paulus MP, Fudge JL, Yang TT, et al. Sucrose activates human taste pathways differently from artificial sweetener. Neuroimage (2008) 39(4):1559-69. doi:10.1016/j.neuroimage.2007. 10.061

119. Eldeghaidy S, Marciani L, McGlone F, Hollowood T, Hort J, Head K, et al. The cortical response to the oral perception of fat emulsions and the effect of taster status. J Neurophysiol (2011) 105(5):2572-81. doi:10.1152/jn.00927. 2010

120. Grabenhorst F, Rolls ET, Parris BA, d'Souza AA. How the brain represents the reward value of fat in the mouth. Cereb Cortex (2010) 20(5):1082-91. doi:10.1093/cercor/bhp169

121. Smeets PA, Weijzen P, Graaf C, Viergever MA. Consumption of caloric and non-caloric versions of a soft drink differentially affects brain activation during tasting. Neuroimage (2011). Available from: http://0-onlinelibrary.wiley.com. library.newcastle.edu.au/o/cochrane/clcentral/articles/805/CN-00780805/ frame.html

122. Grabenhorst F, Rolls ET, Bilderbeck A. How cognition modulates affective responses to taste and flavor: top-down influences on the orbitofrontal and pregenual cingulate cortices. Cereb Cortex (2008) 18(7):1549-59. doi:10.1093/ cercor/bhm 185

123. Bragulat V, Dzemidzic M, Bruno C, Cox CA, Talavage T, Considine RV, et al. Food-related odor probes of brain reward circuits during hunger: a pilot fMRI study. Obesity (Silver Spring) (2010) 18(8):1566-71. doi:10.1038/oby. 2010.57
124. Eiler IWJA, Dzemidzic M, Case KR, Considine RV, Kareken DA. Correlation between ventromedial prefrontal cortex activation to food aromas and cue-driven eating: an fMRI study. Chemosensory Percept (2012) 5(1):27-36. doi:10.1007/s12078-011-9112-6

125. Kemps E, Tiggemann M, Bettany S. Non-food odorants reduce chocolate cravings. Appetite (2012) 58(3):1087-90. doi:10.1016/j.appet.2012.03.002

126. Purnell JQ, Klopfenstein BA, Stevens AA, Havel PJ, Adams SH, Dunn TN, et al. Brain functional magnetic resonance imaging response to glucose and fructose infusions in humans. Diab Obes Metab (2011) 13(3):229-34. doi:10.1111/j.1463-1326.2010.01340.x

127. Burger KS, Stice E. Frequent ice cream consumption is associated with reduced striatal response to receipt of an ice cream-based milkshake. Am J Clin Nutr (2012) 95(4):810-7. doi:10.3945/ajcn.111.027003

128. Stice E, Yokum S, Burger KS, Epstein LH, Small DM. Youth at risk for obesity show greater activation of striatal and somatosensory regions to food. J Neurosci (2011) 31(12):4360-6. doi:10.1523/JNEUROSCI.6604- 10.2011

129. Bohon C, Stice E. Reward abnormalities among women with full and subthreshold bulimia nervosa: a functional magnetic resonance imaging study. Int J Eat Disord (2011) 44(7):585-95. doi:10.1002/eat.20869

130. Bohon C, Stice E. Negative affect and neural response to palatable food intake in bulimia nervosa. Appetite (2012) 58(3):964-70. doi:10.1016/j.appet.2012. 02.051

131. Brooks SJ, Odaly OG, Uher R, Friederich HC, Giampietro V, Brammer M, et al. Differential neural responses to food images in women with bulimia versus anorexia nervosa. PLoS One (2011) 6(7):e22259. doi:10.1371/journal.pone. 0022259

132. Frank GKW, Reynolds JR, Shott ME, O'Reilly RC. Altered temporal difference learning in bulimia nervosa. Biol Psychiatry (2011) 70(8):728-35. doi:10.1016/j.biopsych.2011.05.011

133. Schienle A, Schafer A, Hermann A, Vaitl D. Binge-eating disorder: reward sensitivity and brain activation to images of food. Biol Psychiatry (2009) 65(8):654-61. doi:10.1016/j.biopsych.2008.09.028

134. Weygandt M, Schaefer A, Schienle A, Haynes JD. Diagnosing different bingeeating disorders based on reward-related brain activation patterns. Hum Brain Mapp (2012) 33(9):2135-46. doi:10.1002/hbm.21345

135. Felsted JA, Ren X, Chouinard-Decorte F, Small DM. Genetically determined differences in brain response to a primary food reward. J Neurosci (2010) 30(7):2428-32. doi:10.1523/JNEUROSCI.5483-09.2010

Conflict of Interest Statement: The authors declare that the research was conducted in the absence of any commercial or financial relationships that could be construed as a potential conflict of interest.

Received: 20 February 2014; accepted: 17 June 2014; published online: 09 July 2014. Citation: Pursey KM, Stanwell P, Callister RJ, Brain K, Collins CE and Burrows $T L$ (2014) Neural responses to visual food cues according to weight status: a systematic review of functional magnetic resonance imaging studies. Front. Nutr. 1:7. doi: 10.3389/fnut.2014.00007

This article was submitted to Eating Behavior, a section of the journal Frontiers in Nutrition.

Copyright (C) 2014 Pursey, Stanwell, Callister, Brain, Collins and Burrows. This is an open-access article distributed under the terms of the Creative Commons Attribution License (CC BY). The use, distribution or reproduction in other forums is permitted, provided the original author(s) or licensor are credited and that the original publication in this journal is cited, in accordance with accepted academic practice. No use, distribution or reproduction is permitted which does not comply with these terms. 